\title{
Ultrasensitive TES bolometers for space based FIR astronomy
}

D. Morozov, P. D. Mauskopf, P. Ade, M. Bruijn, P. A. J. de Korte, H. Hoevers, M. Ridder, P. Khosropanah, B. Dirks, and J.-R. Gao

Citation: AIP Conference Proceedings 1185, 48 (2009);

View online: https://doi.org/10.1063/1.3292385

View Table of Contents: http://aip.scitation.org/toc/apc/1185/1

Published by the American Institute of Physics 


\title{
Ultrasensitive TES bolometers for space based FIR astronomy
}

\author{
D. Morozov*, P. D. Mauskopf*, P. Ade*, M. Bruijn ${ }^{\dagger}$, P. A. J.de Korte ${ }^{\dagger}$, H. Hoevers ${ }^{\dagger}$, \\ M. Ridder ${ }^{\dagger}$, P. Khosropanah ${ }^{\dagger}$, B. Dirks ${ }^{\dagger}$ and J.-R. Gao** \\ ${ }^{*}$ Cardiff University, Cardiff, CF24 3AA, UK \\ ${ }^{\dagger}$ SRON, Netherlands Institute for Space Research, Utrecht, 3584, Netherlands \\ ${ }^{* *}$ Delft University of Technology, Delft, 2628, Netherlands
}

\begin{abstract}
We present results from the development of a background limited transition edge sensor (TES) bolometer for the wavelength band $30-60 \mu \mathrm{m}$. The bolometer consists of a Ti/Au superconducting thermometer and a Ta radiation absorber deposited on a $200 \mu \mathrm{m} \times 300 \mu \mathrm{m}$ membrane of $\mathrm{Si}_{\mathrm{x}} \mathrm{N}_{\mathrm{y}}$ suspended on long, narrow legs. This device is voltage biased and the current through the device is measured by a SQUID amplifier. The thermometer has transition temperature $T_{\mathrm{c}}=108 \mathrm{mK}$ and the device is operated from a $70 \mathrm{mK}$ base plate. FIR radiation is coupled into a multimode horn with entrance aperture of $450 \mu \mathrm{m}$, length $4.5 \mathrm{~mm}$ and exit aperture of $45 \mu \mathrm{m}$, which feeds a metal integrating cavity containing the detector. The radiation band is defined by a pair of lowpass and highpass mesh filters in front of the horn. Here we present measurements of optical noise equivalent power (NEP), optical efficiency, dynamic range and time constant. The results show that measured TES detectors are close to meeting the requirement of the "Band 3" of SAFARI FTS imaging instrument [1] on the SPICA mission [2].
\end{abstract}

Keywords: Transition edge sensor, Far-IR detector, $\mathrm{Si}_{x} \mathrm{~N}_{y}$ thermal transport

PACS: $85.25 . \mathrm{Pb}, 95.85 . \mathrm{Gn}, 63.22 .+\mathrm{m}$

\section{INTRODUCTION}

The sensitivity of the current generation of Far-Infrared (FIR) and submillimeter-wave astronomical instruments such as HERSCHEL and SCUBA2 is limited by emission from the warm telescope and/or atmosphere in the case of ground based instruments. Several newly proposed space missions such as SPICA and FIRI include actively cooled telescopes. This would allow FIR observations limited by the photon noise from the astronomical sources themselves and enable detailed spectroscopic measurements of extremely faint sources such as high redshift galaxies and nearby protoplanetary disks. In order to take advantage of these low background platforms, FIR detectors with a low level of noise equivalent power $(N E P)$ are required. The detector requirements for broad band imaging and spectroscopy such as in the Fourier Transform Spectrometer (FTS) proposed for the SAFARI instrument on the SPICA telescope are $N E P=2 \times 10^{-19} \mathrm{~W} / \sqrt{\mathrm{Hz}}$ and time constant $\tau=10 \mathrm{~ms}$. Transition edge superconducting detectors (TES) have recently demonstrated capability of meeting these requirements [3].

\section{DEVICES AND EXPERIMENTAL SETUP}

The TES detectors used for this work were manufactured at SRON/Utrecht. As shown in Figure 1, the bolometers consist of a $1 \mu \mathrm{m}$-thick $\mathrm{Si}_{x} \mathrm{~N}_{y}$ membrane supporting a $100 \times 100 \mu \mathrm{m}^{2} \mathrm{Ti} / \mathrm{Au} / \mathrm{Ti}$ thermometer with $T_{\mathrm{c}}=$ $108 \mathrm{mK}$ and layer thicknesses of 20/55/5 nm. A $200 \times$ $200 \mu \mathrm{m}^{2} \times 7 \mathrm{~nm}$ thick Ta absorber is patterned next to the $\mathrm{Ti} / \mathrm{Au}$ thermometer. The resistance of the absorber is $500 \Omega / \square$. The entire structure is supported by four $\mathrm{Si}_{x} \mathrm{~N}_{y}$ legs, each $240 \mu \mathrm{m}$ long $\times 4 \mu \mathrm{m}$ wide $\times 1 \mu \mathrm{m}$ thick. Niobium wiring is used to contact the thermometer and absorber.

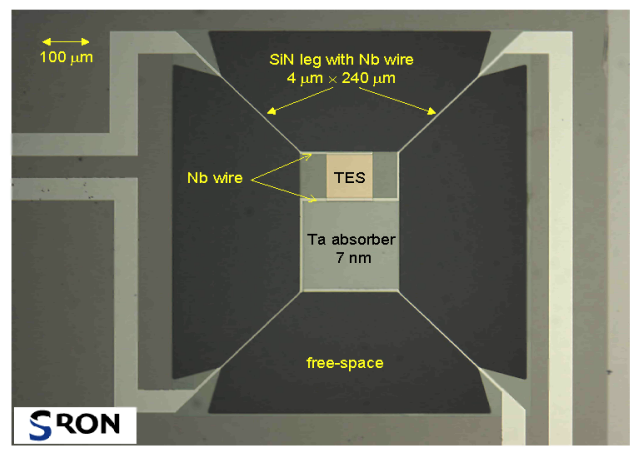

FIGURE 1. TES photograph. 
The test bed is based on a Janis two stage adiabatic demagnetization refrigerator system with base temperature $60 \mathrm{mK}$ which gives us enough margin for TES tests with $T_{\mathrm{c}} \simeq 100 \mathrm{mK}$. Magnetic shielding of the detector volume is achieved with a Metglas ${ }^{(B)} 2705 \mathrm{M}$ foil [4] layer on the first $(1 \mathrm{~K})$ stage shield and $\approx 50 \mu \mathrm{m}$-thick tin-lead alloy shielding on the second $(60 \mathrm{mK})$ stage. The temperature of the cold plate is controlled by a feedback loop which controls the ADR magnet current.

Measurements of the TES were done with a NIST 100-element SQUID series array [5] operated in a fluxlocked-loop using commercial StarCryo [6] read-out electronics adapted to the large output signal voltage from the NIST series arrays. The cold TES circuit is shown in Figure 2, where $R_{\mathrm{S}}$ is the shunt resistor, $R_{\mathrm{b}}$ is the bias resistor, $L_{\mathrm{in}}$ is the input coil of the SQUID and $L_{\mathrm{FB}}$ is the feedback coil of the SQUID. Note that the value $R_{\mathrm{s}}=5 \mathrm{~m} \Omega$ is given at room temperature and $R_{\mathrm{b}}$ is outside the cold part of the cryostat. For IV measurements $R_{\mathrm{b}}=200 \mathrm{k} \Omega$, but we also have to take into account wiring resistance $\approx 400 \Omega$ in series with $R_{\mathrm{b}}$ (for simplicity wiring resistance will be included into the $R_{\mathrm{b}}$ ). We used an Agilent signal generator to provide bias for the detectors.

With extremely low values of the TES bias power $\left(\sim 10^{-15} \mathrm{~W}\right)$ we had to take care that no electomagnetic pick-up affected the measurements. We used RC filters at the output of an RF box mounted on the cryostat wall and an RC filter block with $3 \mathrm{~dB}$ roll-off at $f_{3 \mathrm{~dB}} \simeq 1 \mathrm{MHz}$ at the entrance to the $4 \mathrm{~K}$ volume.

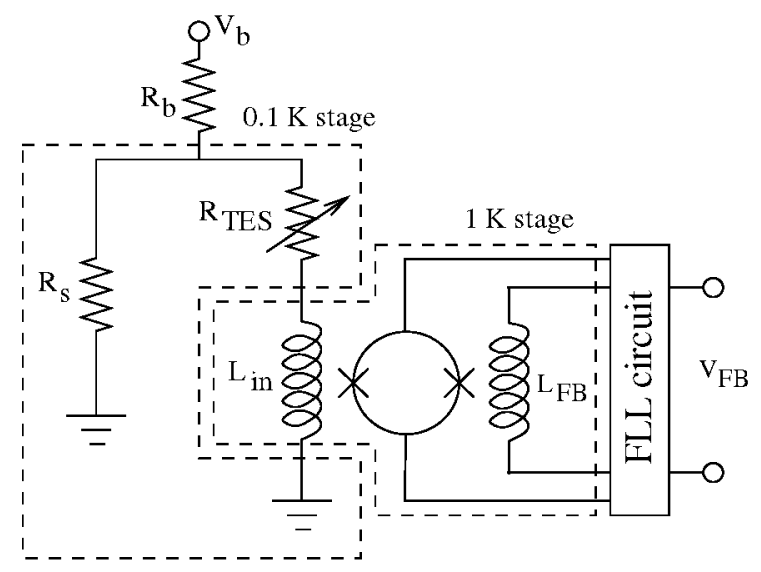

FIGURE 2. TES read out circuit. The SQUID block is mounted on $1 \mathrm{~K}$ stage and connected with TES by means of shielded superconducting $\mathrm{Nb} / \mathrm{Ti}$ cable.

For illumination of the TES we used a variable temperature large area blackbody source mounted on the $4.2 \mathrm{~K}$ shield. The blackbody consists of a $14 \mathrm{~mm}$ diameter copper cone painted on the inside facing the detector with carbon-loaded stycast black paint. A resistive heater and calibrated diode thermometer are glued to the back side of the copper cone and the blackbody assembly is sus- pended on kevlar strings which make it possible to heat it up to $T_{\mathrm{bb}} \sim 30-40 \mathrm{~K}$ with less than $10 \mathrm{~mW}$ of heater power. The thermal stabilization time of the blackbody is appoximately $10-20 \mathrm{~s}$. We also used a smaller area ( $1 \mathrm{~mm} \times 1 \mathrm{~mm}$ ) calibration source with a time constant of approximately $10-20 \mathrm{~ms}$ mounted on the $4.2 \mathrm{~K}$ shield for tests with modulated optical power.

The detector was mounted in a light-tight holder with optical cavity and horn antenna. The frequency band was defined by metal mesh bandpass filters manufactured at Cardiff and similar for those in PACS instrument [7] with cuton frequency $v_{1} \approx 5 \mathrm{THz}$ and cutoff frequency $v_{2} \approx 10 \mathrm{THz}$. We used a system of black baffles to make sure that any leakage of photons onto the shields was minimized.

\section{EXPERIMENTAL RESULTS}

The theoretical performance of a TES bolometer is determined by the thermal conductance, $G$, of the support legs, the heat capacity, $C$, of the absorber and superconducting thermometer, the superconducting transition temperature, $T_{\mathrm{c}}$, and the loop gain of the electrothermal feedback, $L_{0}$ [8]. The phonon-limited sensitivity of a bolometer is given by: $N E P=\sqrt{\gamma 4 k_{\mathrm{B}} T^{2} G}$, where $\gamma$ is a number between 0.5 and 1 depending on the difference between the base temperature, $T_{\mathrm{b}}$, and the transition temperature, $T_{\mathrm{c}}$, of the superconductor and the dependence of the thermal conductance on temperature.

We characterized the TES initially in a dark enclosure to obtain $G(T)$. The power dissipation in the TES, $P_{\mathrm{TES}}=V_{\mathrm{TES}} I_{\mathrm{TES}}$ as a function of base temperature is shown in Figure 3.

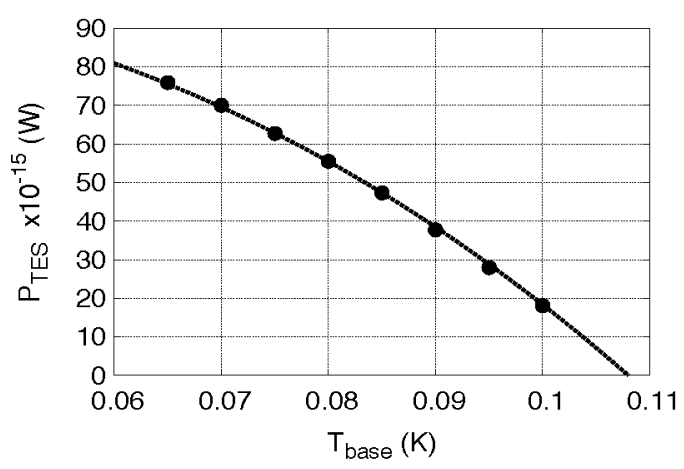

FIGURE 3. Electrical power dissipated in TES vs. base temperature from the IV curves.

The data are well fit by a single power law given by: $P_{\text {TES }}=k\left(T_{\mathrm{c}}^{n}-T^{n}\right)$, where $k$ is a coefficient characterizing heat transfer. The thermal conductance, $G$, of the 
legs is equal to: $G=\mathrm{d} P / \mathrm{d} T=k n T^{n-1}$. After fitting the power vs. temperature data the values shown in Table 1 were calculated for the device. Using the value of $G$ from

TABLE 1. TES parameters

\begin{tabular}{cc}
\hline Parameter & Value \\
\hline $\mathrm{n}$ & 2.5 \\
$\mathrm{k}$ & $2.74 \times 10^{-11}$ \\
$T_{\mathrm{c}}$ & $0.108 \mathrm{~K}$ \\
$\mathrm{G}$ & $2.43 \times 10^{-12} \mathrm{~W} / \mathrm{K}$ \\
\hline
\end{tabular}

Table 1, which was evaluated for $T_{\mathrm{c}}=108 \mathrm{mK}$, we calculate a phonon-limited $N E P=1.3 \times 10^{-18} \mathrm{~W} / \sqrt{\mathrm{Hz}}$. We measured the electrical time constant $\left(\tau_{\mathrm{e} 1}\right)$ by applying a small square wave deviation on top of the DC bias and measuring the resulting current transient. The time constant was computed by fitting an exponential decay to the time stream data. The time constant was measured at different bias points on the IV curve at a base temperature of $70 \mathrm{mK}$ giving values of $\tau_{\mathrm{el}}$ ranging from 1.2 to $2.6 \mathrm{~ms}$.

We performed optical tests with the small volume blackbody to observe a signal from a modulated optical power source. Time traces of the TES signal and blackbody bias voltage are shown in Figure 4 . The current through the TES shown in the trace at the top of Figure 4 decreases exponentially with linear increase of blackbody bias shown at the bottom. This is because the blackbody spectrum for a source at 4-30 K peaks at 0.2-1.5 THz, i.e., at significantly lower frequencies than the minimum in-band frequency of $5 \mathrm{THz}$. Therefore, the in-band power is coming from the Wien tail of the blackbody source spectrum over the entire range of blackbody bias and is exponentially dependent on blackbody temperature.

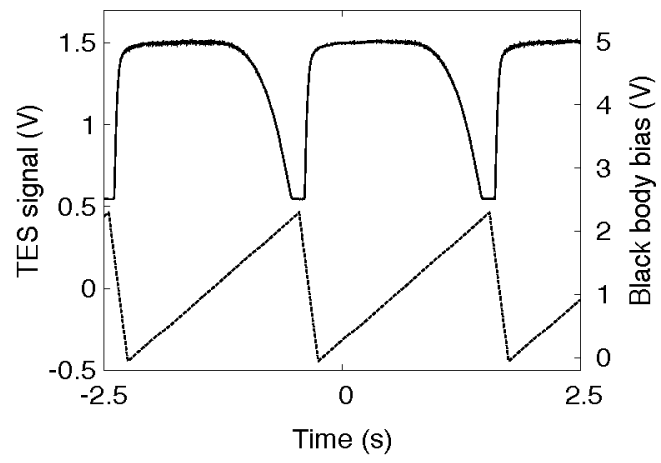

FIGURE 4. Time traces of the TES signal (on the left; upper curve) and the blackbody source bias voltage (on the right; lower curve).

We have measured IV curves of the TES at base temperature $T_{\text {base }}=70 \mathrm{mK}$ with different levels of optical power from the large blackbody source. Power plateaus with different optical load are shown in Figure 5 and the absorbed power in the TES is shown in Figure 6. The smooth curve in Figure 6 shows the amount of power predicted to be incident on the entrance aperture of the detector horn assuming 70\% transmission of the filter stack and a throughput given by $\pi A_{\text {small }}=5 \times 10^{-5} \mathrm{~cm}^{2} \mathrm{sr}$ where $A_{\text {small }}$ is the area of the exit aperture of the collecting horn ( $46 \mu \mathrm{m}$ diameter). The uncertainty in the amount of power incident on the horn is dominated by uncertainties in the blackbody temperature and transmission spectrum of the filters.

At lower blackbody temperatures the absorbed power differs from the estimated emitted power possibly due to stray optical load with power $\leq 6 \times 10^{-16} \mathrm{~W}$. The statistical uncertainty in the measurement of the absorbed power is $<1 \mathrm{fW}$. However, there are systematic errors in the measurement of absorbed power such as variations in the SQUID gain due to different levels of trapped magnetic flux at the level of $\simeq 1 \%$. The optical saturation power for this device was measured to be $P_{\text {sat }} \approx 70 \mathrm{fW}$ at $T_{\mathrm{bb}}=24.9 \mathrm{~K}$ and for $T_{\mathrm{bb}} \geq 25 \mathrm{~K}$ the device was in the normal state.

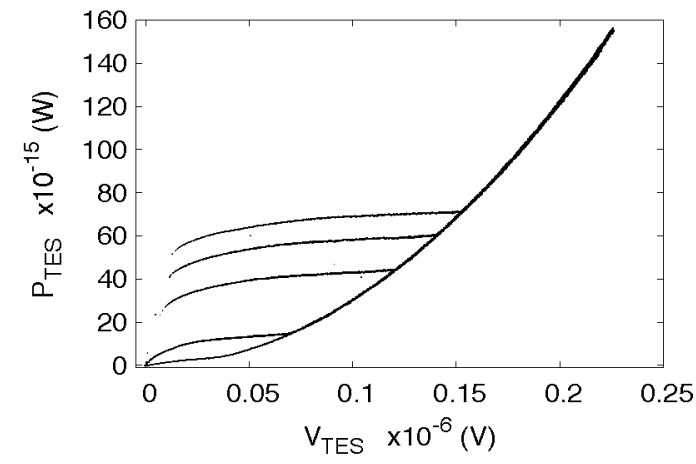

FIGURE 5. Power plateaus at $T_{\text {base }}=70 \mathrm{mK}$ with different radiation loads. Blackbody temperatures from top to bottom: $4.2 \mathrm{~K}, 20.7 \mathrm{~K}, 22.8 \mathrm{~K}, 24.4 \mathrm{~K}, 24.9 \mathrm{~K}$.

We measured the noise spectral density of the TES under different optical loading conditions and use the IV data under the same conditions to derive a noise equivalent power $(N E P)$. The measured $N E P \approx 2 \times$ $10^{-18} \mathrm{~W} / \sqrt{\mathrm{Hz}}$ of the detector with no optical power is shown in Figure 7. The measured spectrum of TES noise with $20 \mathrm{fW}$ detected optical power corresponding to a blackbody temperature of $T_{\mathrm{bb}}=23 \mathrm{~K}$ is shown in Figure 8 . The expected photon shot noise is given by $N E P_{\text {photon }}=\sqrt{2 P_{0} h v}$, where $P_{0}$ is the optical power and $h v$ is the intensity-weighted average photon energy. For our bandpass and $P_{0}=20 \mathrm{fW}$ we calculate $N E P_{\text {photon }}=$ $10^{-17} \mathrm{~W} / \sqrt{\mathrm{Hz}}$ in agreement with the measured noise level at low frequencies. By measuring the frequency de- 


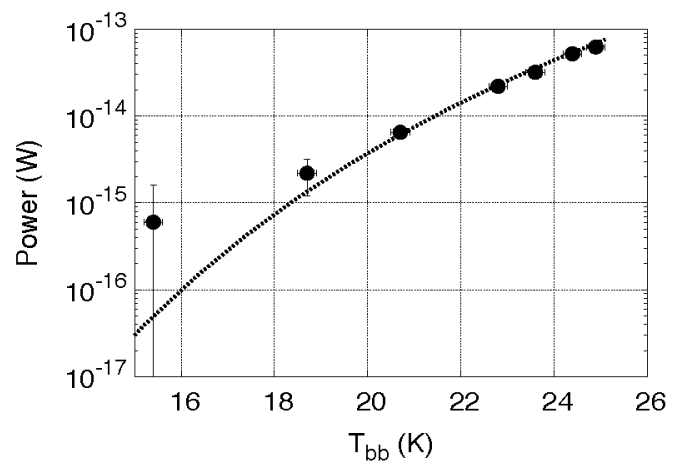

FIGURE 6. Blackbody power absorbed by TES (data points) and estimated blackbody power (line).

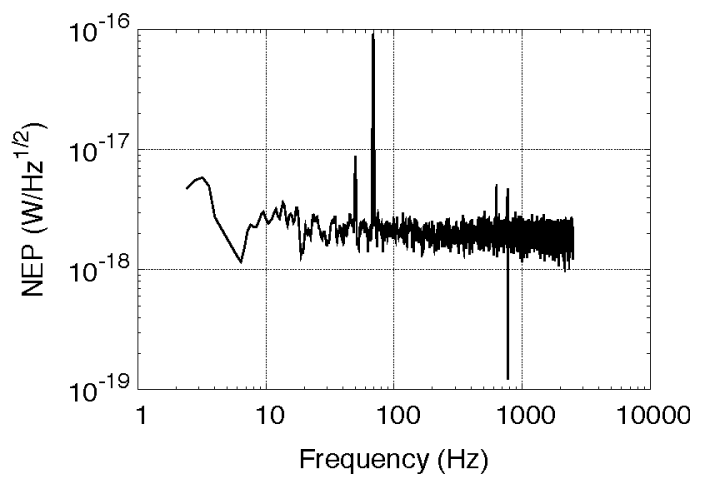

FIGURE 7. Noise spectrum of TES, measured at base temperature $70 \mathrm{mK}$.

pendence of this noise we obtain an estimate of the optical time constant of $\tau_{\text {optical }}=1.5 \mathrm{~ms}$. These measurements can be used as a consistency check of the detector spectral response. If the cut-on frequency of the radiation detected from the cold blackbody was significantly different from $5 \mathrm{THz}$ then we would expect to see a different level of photon noise.

\section{CONCLUSION}

In this paper we presented optical and electrical measurements of a low-G TES detector designed for the frequency band 5-10 THz. The estimated phonon-limited $N E P=1.3 \times 10^{-18} \mathrm{~W} / \sqrt{\mathrm{Hz}}$ and the electrical time constant $\tau_{\mathrm{el}}=1.2-2.6 \mathrm{~ms}$. The measured $N E P \approx 2 \times$ $10^{-18} \mathrm{~W} / \sqrt{\mathrm{Hz}}$ under dark conditions. We also detected both the total power and photon noise from a blackbody

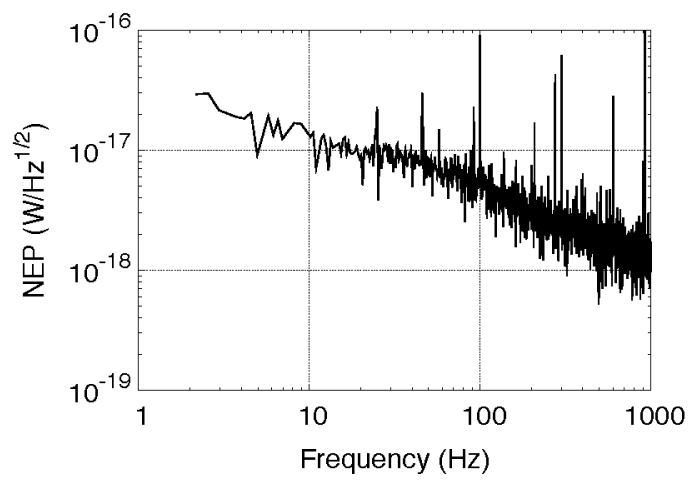

FIGURE 8. Noise spectrum of TES under blackbody illumination with absorbed optical power $P_{0}=20 \mathrm{fW}$, measured at base temperature $65 \mathrm{mK}$.

source at temperatures of approximately $20 \mathrm{~K}$ with high signal-to-noise and from this data derived an optical time constant $\tau_{\text {optical }}=1.5 \mathrm{~ms}$.

\section{ACKNOWLEDGMENTS}

We would like to acknowledge support from the UK Science and Technology Facilities Council (STFC).

\section{REFERENCES}

1. B. Swinyard, "ESI: the far-infrared instrument for the SPICA mission," SPIE, 2006, vol. 6265, p. 62650L.

2. H. Kaneda, T. Nakagawa, T. Onaka, T. Matsumoto, H. Murakami, K. Enya, H. Kataza, H. Matsuhara, and Y. Y. Yui, "Development of space infrared telescope for the SPICA mission," SPIE, 2004, vol. 5487, pp. 991-1000.

3. P. Mauskopf, D. Morozov, D. Glowacka, D. Goldie, S. Withington, M. Bruijn, P. DeKorte, H. Hoevers, M. Ridder, J. V. D. Kuur, and J.-R. Gao, "Development of transition edge superconducting bolometers for the SAFARI far-infrared spectrometer on the SPICA spaceborne telescope," SPIE, 2008, vol. 7020, p. $70200 \mathrm{~N}$, URL http://link.aip.org/link/?PSI/7020/ $70200 \mathrm{~N} / 1$.

4. Metglas inc. (2009), URL http://www. metglas. com.

5. R. Welty, and J. Martinis, Magnetics, IEEE Transactions on 27, 2924-2926 (1991), ISSN 0018-9464.

6. Star cryoelectronics (2009), URL http://www . starcryo.com.

7. Pacs - a photodetector array camera and spectrometer for herschel (2009), URL http: / / pacs . mpe.mpg . de/.

8. K. D. Irwin, and G. C. Hilton, Cryogenic Particle Detection pp. $63-150$ (2005). 